\title{
PREDICTORS OF UNINTENTIONAL POISONING AMONG CHILDREN UNDER 5 YEARS OF AGE IN KARACHI: A MATCHED CASE CONTROL STUDY
}

\author{
B. Ahmed ${ }^{1}$, Z. Fatmi $^{2}$ \\ ${ }^{1}$ Medicine, ${ }^{2}$ Community Health Sciences, Aga Khan University, Karachi, Pakistan
}

Introduction: Poisoning is the $4^{\text {th }}$ leading cause of unintentional injury in children under 5 years of age. The study objective was to determine the factors associated with unintentional poisoning among children under- 5 years of age.

Methods: A matched case- control study was conducted on 120 cases and 360 controls. Children with unintentional poisoning by attending ER physician were included in the study as cases. For each case (3) control children matched for age and gender with diagnosis other than poisoning were selected from the same hospitals ER within 48 hours of case identification. Parents were interviewed by using structured questionnaire containing information on socio-demographic factors, child's behavior, and storage practices of hazardous substances of caregivers inside homes. Conditional logistic regression was performed to analyze the data.

Results: Accessibility to hazardous chemicals and medicines due to unsafe storage (matched odds ratio $=$ 5.6, [95\% CI 1.9,16.7] ), child's behavior reported as usually aggressive ( $\mathrm{mOR}=8.2,[4.6-16.1])$, storage of kerosene oil and petrol in soft drink bottles $(\mathrm{mOR}=3.8,[2.0-7.3])$, low socioeconomic status $(\mathrm{mOR}=9.2$, $[2.8-30.1])$, low mothers education $(\mathrm{mOR}=4.2,[1.8-9.6])$ and history of previous poisoning $(\mathrm{mOR}=8.6$, [1.7 - 43.5] ) were independently related to unintentional poisoning.

Conclusion: The factors are potentially modifiable, key health messages focusing on the safe storage of chemicals and medicines and the use of child resistant containers may play a role in decreasing the burden of childhood poisoning in Karachi, Pakistan. 